\title{
Psicooncología
}

ISSN: 1696-7240

\section{La Activación Conductual (AC) en mujeres con cáncer de mama duran- te la quimioterapia: análisis de la evolución de casos}

\author{
Erica Villoria $^{1 *}$; Concepción Fernández 2 ; Paula Fernández ${ }^{3}$; Ignacio Peláez ${ }^{4}$
}

Recibido: 13 de enero de 2020 / Aceptado: 1 de febrero de 2020

Resumen: Objetivo: Valorar la utilidad de la Activación Conductual para el afrontamiento del cáncer. Método: 18 pacientes con cáncer de mama de reciente diagnóstico siguieron 6 sesiones protocolarizadas dirigidas a recuperar actividades relevantes, eliminar conductas de enfermedad y modificar patrón de evitación experiencial. El análisis funcional determinó objetivos concretos de intervención y la estimación del cambio clínico en cada caso. Se evaluó el cambio pre-post-seguimiento (trimestral). Resultados: Se observa recuperación de las actividades, en particular, domésticas $(\mathrm{p}=0,005)$ y ocio $(p=0,05)$. Es escasa la presencia de patrones de evitación, aunque se logra su reconocimiento. Las conductas de enfermedad se reconocen y reducen $(p=0,03)$. No hay casos de ansiedad ni depresión (HAD), pero sí sintomatología que mejora durante la intervención. Conclusiones: La orientación de los pacientes oncológicos hacia el mantenimiento de actividades de la vida cotidiana relevantes durante la quimioterapia, parece mejorar el afrontamiento de la enfermedad.

Palabras clave: Terapia de Activación Conductual; cáncer; afrontamiento; funcionalidad; estado emocional.

\section{[en] Behavioral Activation Therapy (AC) in women with breast cancer undergoing chemotherapy: case evolution analysis.}

\begin{abstract}
Objective: To assess the utility of Behavioral Activation for coping with cancer. Method: 18 patients with recently diagnosed breast cancer followed 6 protocoled sessions, aimed to recover relevant activities, eliminate illness behavior and to modify experiential avoidance patterns. Functional analysis determined concrete intervention objectives and the estimation of clinically significant change for each case. Pre-post and three months follow up changes were assessed. Results: Activity recovery is observed,
\end{abstract}

1 Erica Villoria Fernández. Universidad Autónoma de Chile, Chile. Instituto de Estudios Sociales y Humanísticos. Chile.

E-mail: erica.villoria@uautonoma.cl

2 Concepción Fernández Rodríguez. Universidad de Oviedo, Oviedo (Asturias), España. Departamento de Psicología. Oviedo, España. CP: 33012

E-mail: frconcha@uniovi.es

3 Paula Fernández García. Universidad de Oviedo, Oviedo (Asturias), España. Departamento de Psicología. Oviedo, España. CP: 33012

E-mail: paula@uniovi.es

4 Ignacio Peláez. Unidad de Oncología Médica, Hospital Universitario de Cabueñes, Cabueñes, Gijón (Asturias), España. CP: 33394

E-mail: ignacio.pelaez@sespa.es

* Dirección de correspondencia: Erica Villoria Fernández. Universidad Autónoma de Chile, Chile. Instituto de Estudios Sociales y Humanísticos. Galvarino Gallardo 1973, $2^{\circ}$ piso, Santiago de Chile (Chile). CP: 8910132. E-mail: erica.villoria@uautonoma.cl 
specifically related to of daily living $(\mathrm{p}=.005)$ and leisure activities $(\mathrm{p}=.05)$. The presence of avoidance patterns is scarce, although recognizable. Illness behaviors are recognized and reduced $(p=.03)$. There were no cases of anxiety and depression (HAD), although some symptomatology is detected, it evolves favorably during the intervention. Conclusions: The guidance of oncologic patients towards maintaining relevant activities of daily living during chemotherapy, seems to improve the coping with the disease.

Keywords: Behavioral Activation Therapy; cancer; coping; functionality; emotional state.

Sumario: 1. Introducción 2. Método 3. Resultado 4. Discusión 5. Agradecimientos 6. Referencias bibliográficas.

Cómo citar: Villoria E, Fernández C, Fernández P, Peláez I. La Activación Conductual (AC) en mujeres con cáncer de mama durante la quimioterapia: análisis de la evolución de casos. Psicooncología 2020;17:11-24. doi: 10.5209/psic.68240.

\section{Introducción}

Estudios sobre la calidad de vida del enfermo oncológico indican como la pérdida de funcionalidad y los cambios en las actividades diarias se relacionan con la aparición de alteraciones emocionales, mayor sintomatología y deterioro en la calidad de vida ${ }^{(1-6)}$. Los problemas que ha de afrontar el paciente oncológico se asocian con las dificultades derivadas de la enfermedad y el tratamiento (síntomas, efectos secundarios, etc.). Ante esta situación, el entorno del enfermo puede suponer un alivio para éste, al menos inicialmente, al delegar responsabilidades $\mathrm{y}$ tareas en allegados y distanciarse de las preocupaciones ${ }^{(7-9)}$. Sin embargo, esto puede conllevar pérdida de funcionalidad que no siempre se justifica por la enfermedad, reduciendo la posibilidad de contacto con aquellas situaciones de valor que formaban parte de la vida del paciente antes del diagnóstico. Todo ello puede desencadenar diferentes problemas emocionales como tristeza, aislamiento, inutilidad, etc. y, en especial, estado de ánimo depresivo, referido frecuentemente en la literatura ${ }^{(10-13)}$.

En este contexto, se pueden analizar los problemas del paciente oncológico desde una perspectiva contextual, tomando como referencia la Terapia de Activación Conductual (AC) $)^{(14-16)}$. La AC nace orientada a la depresión y tiene su base en las terapias conductuales tradicionales poniendo el énfasis en las circunstancias personales que mantienen la conducta. Propone que las conductas que la persona pone en marcha ante un problema pueden ser el obstáculo en vez de la solución. Así, introduce el concepto de evitación conductual, entendiendo que el uso de estrategias de evitación ante un problema sitúa a la persona en una actitud pasiva, dificultando el contacto con las condiciones de las que podría depender una mejoría. Se propone entonces un modelo de aceptación-cambio enseñando a la persona a formular y lograr objetivos conductuales a pesar del estado de ánimo negativo, entendiendo que, en la medida en que se produzca un incremento de la conducta, éste puede mejorar ${ }^{(17)}$. La lógica entonces desde la $\mathrm{AC}$ para los pacientes oncológicos sería lograr el compromiso con el curso de su vida, involucrándose en aquellas actividades (rutinas, gratificantes) capaces de activar sus fuentes de reforzamiento cotidianas 
(activación), eliminar conductas de enfermedad (evitación) y mantener conductas saludables, logrando un mejor afrontamiento a la enfermedad y la prevención de alteraciones emocionales ${ }^{(11)}$.

La propuesta de la AC para los problemas del paciente oncológico cobra más relevancia si se tienen en cuenta algunas de las limitaciones presentadas por las distintas intervenciones psicológicas que desde otras orientaciones (cognitivo-conductual, educativas, etc.) se han puesto en marcha en el ámbito de la oncología ${ }^{(18,19)}$. Aunque la literatura señala los efectos favorables de estas en distintos aspectos del paciente (estado emocional, síntomas, funcionalidad, calidad de vida) ${ }^{(20,21,22)}$, en muchas se han aplicado intervenciones basadas en paquetes de técnicas estandarizados, generando una falta de especificidad entre técnicas y objetivos adecuados para cada paciente y la dificultad para identificar en qué condiciones y para qué pacientes tienen mayor utilidad $^{(23,24)}$. Esto señala la necesidad de un planteamiento de intervención que, como la $\mathrm{AC}$, se muestre flexible y orientado a los objetivos relevantes para la persona, haciéndola idónea para superar estas limitaciones.

Por todo lo anterior y, sumando la eficacia demostrada de la AC en la depresión ${ }^{(25-29)}$ y, más incipientemente en el ámbito de la oncología $a^{(7,8,11,12,30-34)}$, se propone implementar una intervención basada en la $\mathrm{AC}$, siguiendo trabajos previos ${ }^{(8,11)}$ y $\sin$ presuponer la presencia de psicopatología en los pacientes oncológicos, para valorar su utilidad en el afrontamiento a la enfermedad oncológica.

\section{Método}

\section{Participantes}

En el estudio participaron 33 pacientes con cáncer de mama, seleccionados en la primera cita en Oncología Médica del Hospital de Cabueñes de Gijón (Asturias, España) independientemente del estadio inicial de la enfermedad y cuando el tratamiento oncológico a seguir fue la quimioterapia. Los pacientes fueron informados de los objetivos y procedimiento del estudio, dando su consentimiento para participar.

Para el análisis de casos se tuvieron en cuenta 18 participantes que finalizaron la intervención y contaron con un seguimiento a los tres meses, garantizando así la obtención de datos completos sobre su evolución. La pérdida de participantes se justifica por su estado físico, el ritmo asistencial del servicio y abandonos voluntarios.

Las características sociodemográficas y clínicas de la muestra de estudio se exponen en la Tabla 1, cuya comparación estadística con la muestra inicial no señala diferencias significativas. 
Tabla 1. Datos sociodemográficos y clínicos de la muestra

\begin{tabular}{|c|c|c|}
\hline & $\begin{array}{l}\text { Muestra final } \\
\mathrm{N}=18\end{array}$ & $\begin{array}{l}\text { Muestra inicial } \\
\mathrm{N}=33\end{array}$ \\
\hline Edad (M/ DT) & $49,22 / 12,70$ & $52,57 / 12,48$ \\
\hline $\mathrm{N}^{\mathrm{o}}$ de hijos (M/DT) & $1,33 / 1,02$ & $1,58 / 1,17$ \\
\hline $\mathrm{N}^{\circ}$ de familiares convive (M/DT) & $1,66 / 0,97$ & $1,61 / 1,06$ \\
\hline $\begin{array}{l}\text { Estado Civil (\%) } \\
\text { Soltero } \\
\text { Casado/pareja } \\
\text { Viudo } \\
\text { Separado }\end{array}$ & $\begin{array}{l}22,2 \\
61,1 \\
5,6 \\
11,1\end{array}$ & $\begin{array}{l}12,1 \\
60,6 \\
12,1 \\
15,2\end{array}$ \\
\hline $\begin{array}{l}\text { Estudios (\%) } \\
\text { Sin estudios } \\
\text { Primarios } \\
\text { Secundarios } \\
\text { Universitarios }\end{array}$ & $\begin{array}{l}- \\
33,3 \\
44,4 \\
22,2\end{array}$ & $\begin{array}{l}- \\
43,8 \\
34,4 \\
21,9\end{array}$ \\
\hline $\begin{array}{l}\text { Actividad Laboral (\%) } \\
\text { Desempleo } \\
\text { Activo } \\
\text { Baja médica } \\
\text { Ama de casa } \\
\text { Jubilado }\end{array}$ & $\begin{array}{l}16,7 \\
- \\
55,6 \\
27,8 \\
-\end{array}$ & $\begin{array}{l}12,1 \\
6,1 \\
45,5 \\
24,2 \\
12,1\end{array}$ \\
\hline $\begin{array}{l}\text { Estadios (\%) } \\
\text { I } \\
\text { II-A } \\
\text { II-B } \\
\text { III } \\
\text { III-A } \\
\text { III-B } \\
\text { III-C } \\
\text { IV }\end{array}$ & $\begin{array}{l}16,7 \\
27,8 \\
33,3 \\
11,1 \\
- \\
5,6 \\
5,6 \\
-\end{array}$ & $\begin{array}{l}18,2 \\
24,2 \\
21,2 \\
6,1 \\
15,2 \\
3,0 \\
6,1 \\
6,1\end{array}$ \\
\hline $\begin{array}{l}\text { Tratamiento previo }(\%) \\
\text { No } \\
\text { Cirugía conservadora de mama } \\
\text { Cirugía radical de mama }\end{array}$ & $\begin{array}{l}11,1 \\
50,0 \\
38,9\end{array}$ & $\begin{array}{l}9,1 \\
45,5 \\
45,5\end{array}$ \\
\hline
\end{tabular}

\section{Instrumentos}

1) Protocolo (ad hoc): recoge datos sociodemográficos, sobre la enfermedad y el tratamiento médico. Es cumplimentado en la primera consulta por el oncólogo y el psicólogo.

2) Escala de Ansiedad y Depresión Hospitalaria (HAD) ${ }^{(35)}$ : evalúa el estado emocional de sujetos atendidos en servicios hospitalarios de consulta externa no psiquiátricos. Con el fin de evitar falsos positivos en la evaluación psicopatológica 
en estos contextos, se excluyen referencias a síntomas físicos. La escala consta de 14 ítems divididos en dos subescalas, ansiedad (HAD-A) y depresión (HAD-B), que son valoradas en función de los criterios propuestos por Zigmond y Snaith ${ }^{(35)}$. puntuación de 0 a 7, ausente de síntomas; de 8 a 10, caso dudoso; y de 11 a 21, caso de depresión y/o ansiedad. Diferentes estudios indican sus adecuadas propiedades psicométricas $^{(36-39)}$. Es cumplimentada en todos los momentos de medida por el paciente.

3) Entrevista semiestructurada ${ }^{(40)}$ : utilizada en cada sesión y diseñada ad hoc para la valoración de: (a) Funcionalidad/actividades relevantes: actividad laboral, cuidados personales, actividades domésticas, actividades de ocio, relaciones familiares, relaciones de pareja, relaciones sexuales y relaciones con amigos. Se registra de 0 a 1 el nivel de funcionalidad en cada ámbito durante el periodo entre sesiones de tratamiento $(0=\sin$ actividad; $1=$ no mantiene; $2=$ mantiene $)$. Se evalúan en todas las sesiones de tratamiento y en el seguimiento. (b) Presencia/ ausencia y reconocimiento de conductas de enfermedad (si/no). (c) Presencia/ ausencia y reconocimiento de patrones de evitación de actividades relevantes y gratificantes (si/no). (d) Presencia/ausencia y reconocimiento de pensamientos y emociones de evitación con relación a la enfermedad y/o tratamiento (si/no). Las condiciones b, c y d son registradas desde la segunda sesión por ser información específica de la intervención psicológica.

\section{Procedimiento}

La intervención se organizó en seis sesiones individuales de 45 minutos aproximadamente, coincidiendo con la administración de cada ciclo de quimioterapia. Fue integrada en la rutina médica para provocar la menor interferencia en la vida cotidiana del paciente. Además, se llevó a cabo un seguimiento a los tres meses de finalizar la quimioterapia.

El protocolo de intervención ${ }^{(11)}$, adaptado a las necesidades de cada caso, tuvo por objetivos específicos la recuperación y/o mantenimiento de las actividades relevantes y gratificantes de la vida del paciente; la eliminación de conductas de enfermedad aprendidas y la modificación de patrones de evitación (pensamientos/emociones de evitación y evitación de actividades relevantes).

En la primera sesión, se pretendía identificar problemas y limitaciones que pudieran estar ocasionando malestar emocional y alteraciones en el funcionamiento cotidiano. Se evaluaron los repertorios de conducta saludables y las fuentes de reforzamiento de las que disponía el paciente. Esta información serviría como medida pretratamiento (línea base) y para establecer los objetivos para la intervención.

En cada sesión, el terapeuta trataba de enseñar al paciente a observar sus conductas mediante la descripción en términos contextuales de sus rutinas, emociones y estado físico y, a reconocer las contingencias (ambientales y privadas pensamientos/ emociones) implicadas es su fortalecimiento o debilitamiento (descripción/análisis funcional de la conducta). Se trataba de identificar los comportamientos disfuncionales con relación al afrontamiento de la enfermedad. Se evaluaban repertorios de conducta saludables y las fuentes de reforzamiento de las que disponía. Se pretendía fomentar comportamientos reforzados naturalmente e incrementar la sensibilidad del sujeto a las contingencias ambientales $\mathrm{y}$, alternativamente, eliminar conductas de enfermedad y modificar patrones de evitación. 
Las metas de la intervención para cada participante respondían a los objetivos de éste, en función de su vida y su entorno. Mediante su compromiso (y allegados) se perseguía movilizar los recursos del paciente y/o lograr el cambio en las contingencias del entorno necesario para poner en marcha o activar los comportamientos saludables a pesar de la enfermedad. Se trataba de establecer metas orientadas a mantener las actividades relevantes/gratificantes de su vida. Además, ante la presencia de posibles condiciones (bien de la enfermedad/otros) no modificables se trabajaba su aceptación para favorecer la consecución de objetivos.

En la intervención, además del análisis funcional de la conducta (fundamental para determinar metas y tareas para cada caso y en cada sesión) ya señalado, se emplearon diferentes técnicas en función de las necesidades del caso. Así, con el objetivo de que el paciente identificara las condiciones de las que dependía su conducta y estableciera relaciones entre el hacer, el sentir y su entorno, éste cumplimentaba registros de actividad y diarios de pensamientos, aplicando ensayo de conducta y modelado para lograr su congruencia. Para la recuperación y/o mantenimiento de las actividades relevantes/gratificantes, mediante jerarquías de actividad, se trabajaba con el paciente la programación de actividades para fijar objetivos viables y relevantes, para que su realización se ajustara a las claves contextuales que podían llegar a controlar la conducta asegurando, además, una ejecución exitosa y con mayor probabilidad de ocurrencia en el futuro. Los contratos de contingencias y el entrenamiento en habilidades facilitaban el mantenimiento y/o recuperación de actividades, poniendo las conductas bajo el control de las claves del contexto y resolviendo posibles conflictos que pudieran favorecer conductas de enfermedad o evitación. Por último, para la aceptación de la situación de enfermedad y eliminar pensamientos y patrones de evitación se utilizaban metáforas, ejemplos y paradojas para facilitar el proceso.

Toda la investigación, aprobada por el Comité Ético de la Institución, se realizó cumpliendo los estándares éticos de la APA para el tratamiento de humanos.

\section{Análisis de datos}

Para examinar el cambio clínico (pretratamiento, postratamiento y seguimiento) se realizó el estudio descriptivo (frecuencias y porcentajes) de sujetos que mantenían la funcionalidad en la condición/variable analizada. La significación del cambio clínico se estableció a partir del concepto de "validación social" en relación, específicamente, a que los cambios producidos por la intervención psicológica correspondieran con los objetivos del tratamiento suponiendo, además, una mejor adaptación en un aspecto importante de la vida del paciente ${ }^{(41)}$. También, se calcularon las puntuaciones medias en ansiedad y depresión evaluadas mediante el HAD en cada momento de medida.

Para examinar el cambio estadístico a lo largo de tres momentos (pretratamiento, postratamiento y seguimiento) en las variables objetivo se empleó la prueba Friedman en el caso del HAD, puesto que, aunque se cumplían los supuestos paramétricos el tamaño de muestra era reducido, y la prueba no paramétrica de Cochran (Q) en las siguientes condiciones: mantenimiento de la actividad diaria (doméstica, ocio, sexual y actividades gratificantes); presencia/ausencia de patrones de evitación de las actividades relevantes y su reconocimiento; presencia/ausencia de pensamientos/ emociones de evitación y su reconocimiento. Presencia/ausencia de conductas de enfermedad y su reconocimiento.

El paquete estadístico utilizado fue el SPSS 25.0 para Windows. 


\section{Resultados}

- Estudio descriptivo para examinar el cambio clínico:

En relación con el estado emocional, en la subescala HAD-a las puntuaciones medias en todos los momentos de medida se mantuvieron en torno a 5-6, por debajo del punto de corte clínico para caso clínico de ansiedad. Se observa como la puntuación inicial es más elevada que en el resto de las medidas $(M=6,16)$, que se mantienen bastante constantes, con un incremento en el fin del tratamiento de quimioterapia, siendo la puntuación media más elevada $(\mathrm{M}=6,26)$. Con respecto a las puntuaciones medias en la subescala HAD-d, en todos los momentos se hallan en torno 4-5, también por debajo del punto de corte clínico, con una puntuación más elevada al finalizar la quimioterapia $(\mathrm{M}=5,73)$. (Tabla 2).

Tabla 2. Puntuaciones medias obtenidas en el HAD

(subescalas de ansiedad, depresión y total).

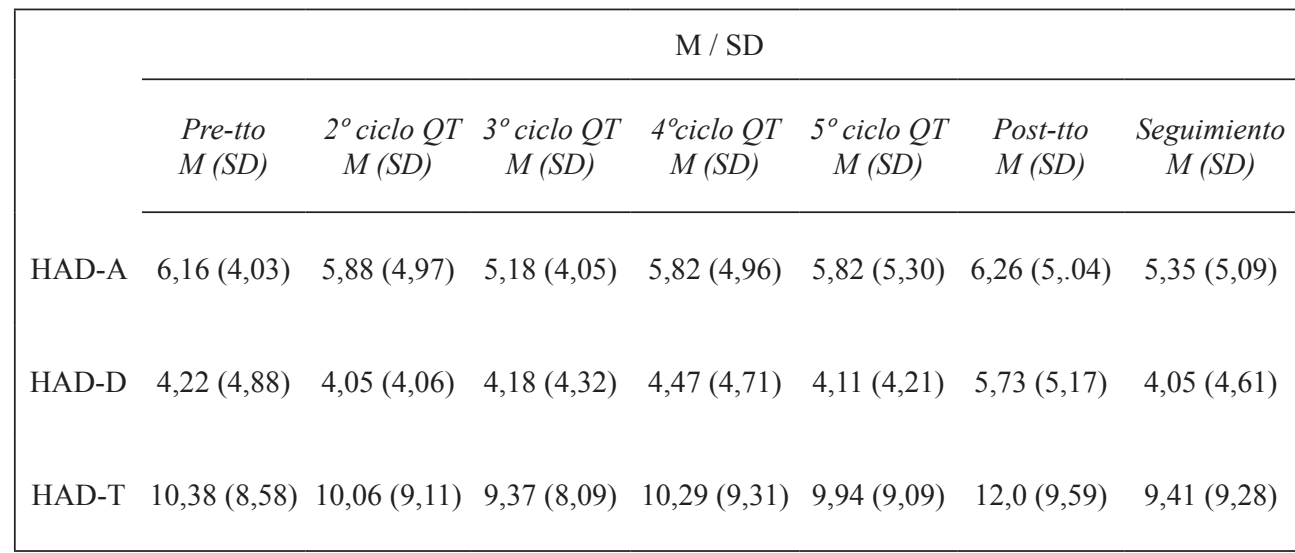

Qt: quimioterapia

Tto: tratamiento

En nivel de funcionamiento, en aquellos ámbitos de la vida (doméstico, ocio, sexual y actividades gratificante) que fueron objeto de la intervención psicológica, a lo largo de las sesiones se observa como en todos ellos se logra el mantenimiento y/o la recuperación de actividad (Figura 1). Se destaca el doméstico donde se observa un incremento del 55,6\%, lo que supone que de 7 pacientes que mantenían la actividad al inicio, 10 más la recuperan al seguimiento. En el ocio se observa un incremento del $38,9 \%$. En el inicio y final, la mitad de las pacientes mantuvieron la actividad. La actividad sexual y actividades gratificantes presentan incrementos menores con respecto al seguimiento (11,1\% y $15,7 \%$, respectivamente). Entre el pretratamiento y el postratamiento se mantienen la actividad sexual y disminuyen levemente las actividades gratificantes.

En los otros ámbitos evaluados no se observaron cambios a lo largo de las medidas. Sólo el ámbito laboral resultó afectado en aproximadamente la mitad de las pacientes, afectación que se mantuvo constante durante la intervención. 
Figura 1. Cambio clínico y estadístico (Prueba Q de Cochran; ${ }^{*} \mathrm{p}<.05$ ) del porcentaje de pacientes que mantiene las actividades relevantes en los momentos de medida analizados (pretratamiento, postratamiento y seguimiento).

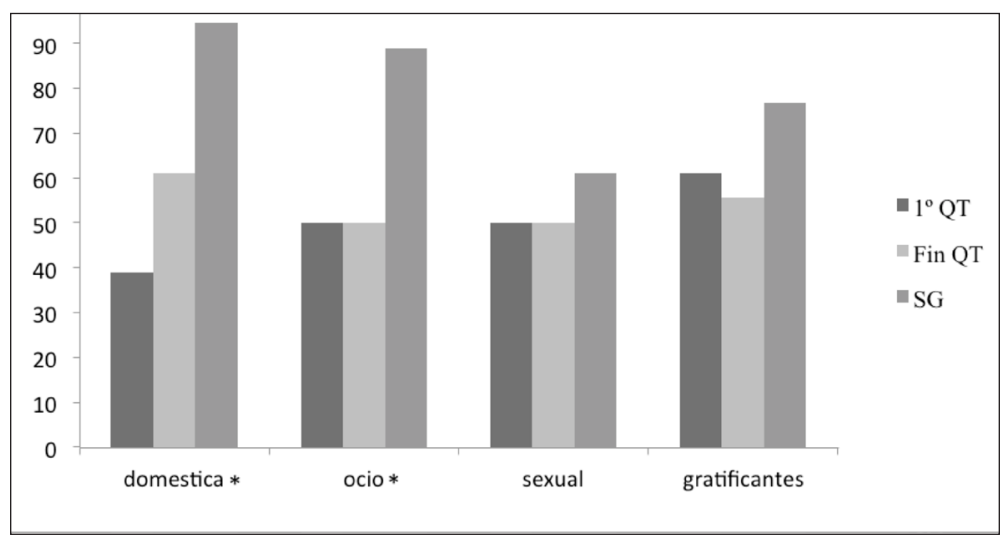

Con respecto a las conductas de enfermedad, patrones de evitación y pensamientos/ emociones de evitación se observó una baja presencia, menos del 30\% de los pacientes. Para las conductas de enfermedad y patrones de evitación se logra el reconocimiento en más del $50 \%$ de los pacientes y, en torno al $40 \%$ en pensamientos/ emociones de evitación. (Figura 2)

Figura 2. Cambio clínico y estadístico (Prueba Q de Cochran; ${ }^{*} p<0,05$ ) del porcentaje de pacientes que presentan y reconocen conductas de enfermedad, patrones y prensamientos/ emociones de evitación en los momentos de medida analizados (pretratamiento, postratamiento y seguimiento).

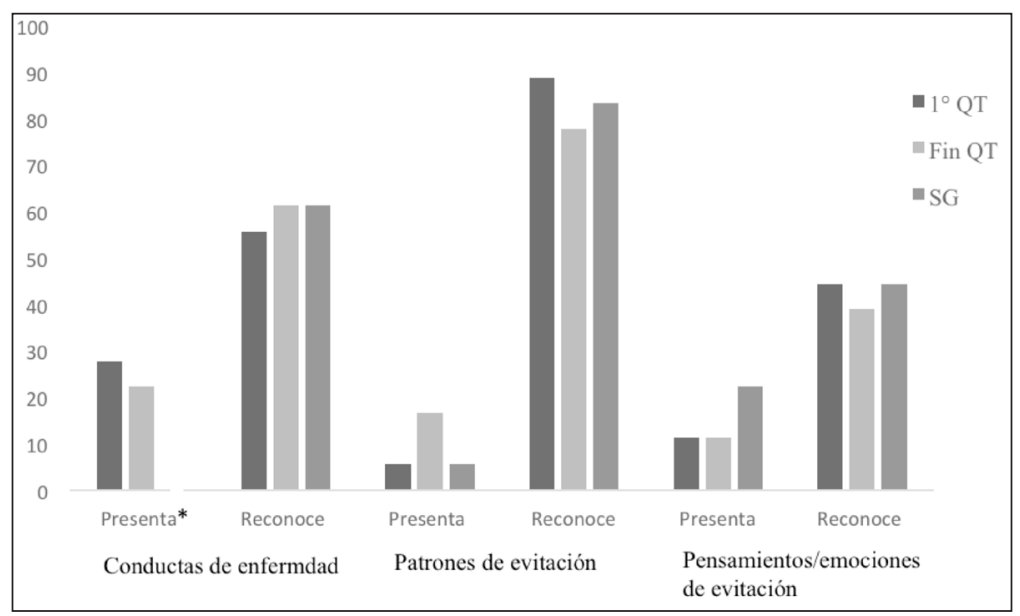

- Estudio estadístico del efecto de la intervención psicológica mediante la evaluación del cambio experimentado en el pretratamiento, postratamiento y seguimiento: 
El análisis estadístico de las puntuaciones del HAD, no indica diferencias significativas a lo largo de las medidas.

En el nivel de funcionamiento, en todos los ámbitos objetivo de la intervención hay una tendencia a su recuperación, especialmente en el seguimiento. No obstante, sólo la actividad doméstica $\left(\mathrm{Q}_{(2)}=10,71 ; p=0,005\right)$ y de ocio $\left(\mathrm{Q}_{(2)}=6 ; p=0,05\right)$ fluctúan de modo significativo a lo largo del tiempo. (Figura 1)

Con respecto a la presencia de conductas de enfermedad, patrones de evitación y pensamientos/emociones de evitación se observa un cambio estadísticamente significativo sólo en la presencia de conductas de enfermedad $\left(\mathrm{Q}_{(2)}=7 ; p=\right.$ $.03)$, que se reducen al final y desaparecen en el seguimiento. Con respecto al reconocimiento de las tres condiciones, no se observan cambios con significación estadística (Figura 2).

\section{Discusión}

El objetivo de este estudio fue valorar la utilidad de una intervención psicológica basada en la AC para el afrontamiento en pacientes con cáncer de mama, siguiendo estudios previos que sugieren su adecuación ${ }^{(7,8,11)}$. Para ello se analizó, estadística y clínicamente, el cambio experimentado de aquellas pacientes que finalizaron la intervención y un seguimiento en las variables objetivo de la misma.

En cuanto a la funcionalidad, se observa el mantenimiento y recuperación de la actividad en los distintos ámbitos relevantes de la vida, a pesar de la posible presencia de los efectos derivados de la quimioterapia (nauseas/vómitos, cansancio, dolor, etc.) que se han relacionado con pérdida de actividad, malestar emocional y peor calidad de vida ${ }^{(42-44)}$. Parecen lograrse los objetivos de la intervención, a pesar de que los síntomas podrían ser un obstáculo para la activación. En esta línea, estudios previos ya indican como intervenciones basadas en la AC logran la recuperación de las actividades durante el tratamiento oncológico a pesar de la elevada sintomatología presente por la quimioterapia ${ }^{(11,8)}$. No obstante, se debe señalar que la mayor recuperación se da en el seguimiento, lo que podría asociarse a la desaparición de los efectos de la quimioterapia. A pesar de esto, aunque no siempre hay recuperación de actividad, sí se logra el mantenimiento de ésta durante el tratamiento en la mayoría de los ámbitos trabajados, lo que también es un objetivo de la intervención psicológica.

Estos resultados con relevancia clínica sólo resultan estadísticamente significativos para la actividad doméstica y de ocio que son, además, los más afectados en la mayoría de las pacientes ya desde el inicio. Esto puede relacionarse con la propia condición de enfermedad en la medida que el entorno familiar y médico pueden favorecer mayor abandono de estos ámbitos, con el objetivo de que el paciente se concentre en su cuidado y recuperación. El logro de objetivos a pesar de la presencia de estos aspectos, que pueden obstaculizar la activación conductual, fortalece la hipótesis del papel positivo de la intervención psicológica aplicada. Además, el ámbito doméstico es el más favorecido al final y, no sólo al seguimiento, lo que también podría destacar el efecto de la intervención psicológica si se tienen en cuenta las características sociodemográficas de la muestra. La mayoría son mujeres casadas que, en general, siguen asumiendo la mayor carga de responsabilidades familiares y del hogar, por lo que activación conductual en 
este ámbito puede resultar más relevante para ellas por el deseo o necesidad de mantener esta actividad como ya se ha presentado en estudios previos ${ }^{(8,11)}$.

Con relación a los patrones y pensamientos/emociones de evitación, aunque la mayoría de los pacientes no parecen presentarlos, se logra un reconocimiento significativo de los mismos. Además, con respecto a las conductas de enfermedad no sólo se observa este reconocimiento, sino que, también se logra su reducción significativa. Estos resultados son consistentes con los objetivos de la intervención psicológica y, además, apoyan la recuperación y mantenimiento de las actividades relevantes, ya que desde la intervención no sólo se pretende eliminar conductas de enfermedad sino, también, alternativamente promover estas actividades y fomentar el afrontamiento al cáncer mediante conductas saludables.

En el estado de ánimo, la presencia de patología clínica parece baja, aunque se aprecia sintomatología. Esto concuerda con estudios previos que indican cifras bajas de ansiedad y depresión con significación clínica en estos pacientes ${ }^{(11,45,46)}$. Durante el tratamiento se observa mejoría, aunque sin evidencia estadística, lo que puede relacionarse con la recuperación de las actividades, por tanto, ir acorde con la lógica de la intervención. En esta línea, diversos estudios han relacionado las alteraciones emocionales con los efectos de la quimioterapia y, también, con las alteraciones de la vida generadas por la situación de enfermedad ${ }^{(47,49-51)}$. Se ha señalado como la recuperación de actividad se asocia a mejores valoraciones del estado de ánimo, aunque la relación entre ambas resulte compleja de determinar ${ }^{(2,6,11,52,53)}$.

Por otro lado, se aprecia mayor sintomatología emocional al inicio y final de la quimioterapia lo que va en consonancia con los momentos destacados en la literatura por sus características. El inicio de la quimioterapia, por la ansiedad asociada a la incertidumbre ante los posibles efectos secundarios y la eficacia del tratamiento ${ }^{(54,55)}$. Y, los momentos finales, por la ruptura con los controles y la atención médica y el miedo al futuro $^{(56,57,58)}$. Esto, por su parte, podría explicar la ausencia de cambios estadísticamente significativos entre el inicio y el final del tratamiento, a pesar de apreciarse mejorías emocionales durante el mismo. Además, destacaría la importancia de abordar específicamente desde la intervención psicológica estas fases de la enfermedad.

En general, parece que la evolución de estas pacientes se muestra favorable en la relación a los objetivos de la intervención. No obstante, estos resultados deben ser tomados con precaución. Por un lado, el reducido número de pacientes no permiten obtener datos robustos para garantizar la generalización de los resultados. Además, en algunas variables y momentos de medida se pierde información porque las condiciones valoradas no afectan en ese momento, lo que limita el uso de análisis estadísticos, haciendo necesario valorar aspectos exclusivamente descriptivos. También, la ausencia de grupo control podría comprometer la utilidad de la intervención psicológica al no poder descartarse que los potenciales efectos terapéuticos de la activación puedan atribuirse a otras condiciones (atención, escucha activa, empatía, etc.), lo cual lejos de ser inocuo, podría suponer una situación de expresión de emociones y, por tanto, tener un efecto terapéutico. No obstante, este estudio pertenece a una serie de investigaciones más amplias que sí contaron con grupo control, el cual era equivalente al grupo de intervención en características clínicas y sociodemográficas y, donde se obtuvieron resultados que apoyan el efecto positivo del protocolo de intervención psicológica ${ }^{(8,11,)}$. Estas investigaciones han mostrado un procedimiento donde el grupo control recibe el mismo número de sesiones que el experimental, orientadas exclusivamente a la evaluación sin proporcionar atención diferencial a las competencias/déficit de los sujetos 
en relación al afrontamiento e la enfermedad. Esto equipara a ambos grupos en cuanto al posible efecto terapéutico derivado de la oportunidad de expresar emociones a una audiencia especializada. Así, mejores resultados en el grupo experimental indicarían un efecto terapéutico derivado de los componentes específicos de la intervención psicológica aplicada y, más allá del posible efecto de la atención dispensada por un psicólogo.

A modo de conclusión, sin perder de vista las limitaciones ya presentadas, parece que la intervención psicológica basada en el procedimiento de la AC favorece el afrontamiento a la enfermedad al lograr promover la activación comportamental de las pacientes mediante el compromiso con su vida, actuando conforme a las cosas valiosas e importantes para ellas. Esta terapia puede llevarse a cabo de una manera estructurada, facilitando su integración en la rutina médica y, a su vez, de forma flexible ajustada a las necesidades individuales y a los objetivos relevantes y condiciones de cada paciente. No obstante, se destaca la necesidad de indagar más en las peculiaridades y características de los pacientes que podrían estar mediando en el efecto y adecuación de la intervención, de manera que ésta pueda mostrarse más eficaz.

\section{Agradecimientos:}

Este estudio se llevó a cabo gracias al apoyo y colaboración de los profesionales del Hospital Universitario de Cabueñes de Gijón (SESPA), España.

\section{Referencias bibliográficas}

1. Decker K, Moineddin R, Kendell C, Urquhart R, Biswanger N, Groome P, et al. Changes in primary care provider utilization by phase of care for women diagnosed with breast cancer: A CanIMPACT longitudinal cohort study. BMC Fam Pract 2019; 20(161). doi: 10.1186/s12875-019-1052-2

2. Faller H, Strahl A, Richard M, Niehues C, Meng K. Symptoms of depression and anxiety as predictors of physical functioning in breast cancer patients. A prospective study using path analysis. Acta Oncol 2017; 56: 1677-81. doi: 10.1080/0284186X.2017.1333630

3. Fernández C, Padierna C, Amigo I, Gracia JM, Fernández R, Peláez I. Calidad de vida informada en pacientes oncológicos paliativos. Index Enferm 2006; (52-53):30-4.

4. Green CR, Hart-Johson T, Loeffler DR. Cancer-related chronic pain: examining quality of life in diverse cancer survivors. Cancer 2011;117:1994-2003. doi: 10.1002/ cncr.25761

5. Nokolić S, SIlić-Stosović D, Kolarevic I, Djurdjević A, Ilić S, Djuričić M. Social participation of women with breast cancer. Vojnosanit Pregl 2015;72:148-54. doi: 10.2298/VSP1502148N

6. Patsou ED, Alexias GT, Anagnostopoulos FG, Karamouzis MV. Physical activity and sociodemographic variables related to global health, quality of life, and psychological factors in breast cancer survivors. Psychol Res Behav Manag 2018;11:371-81. doi: 10.2147/PRBM.S170027

7. Fernández C, Villoria E, Fernández P, González S. Efectos de la Activación Conductual en la calidad de vida y estado emocional de los pacientes con cáncer de pulmón. Psicooncología 2014;11:199-215. doi: 10.5209/rev_PSIC.2014.v11.n2-3.47383 
8. Fernández C, Villoria E, Fernández P, González S, Pérez M. Effects of behavioral activation on the quality of life and emotional state of lung cancer and breast cancer patients during chemotherapy treatment. Behav. Modificat 2019; 43:151-80. doi: $10.1177 / 0145445517746915$

9. Muñoz F, Espinosa JM, Portillo J, Rodríguez G. La familia en la enfermedad terminal. Medicina de Familia 2002;3:190-9.

10. Burgess C, Cornelius V, Love S, Graham J, Richards M, Ramirez A. Depression and anxiety in women with early breast cancer: five-year observational cohort study. BMJ 2005;330:702. doi: 10.1136/bmj.38343.670868.D3

11. Fernández C, Villoria E, Amigo I, Padierna C, Gracia JM, Fernández R et al. Terapia de activación conductual en pacientes con cáncer. Anal Psicol 2011;27:278-91.

12. Hopko DR, Bell JL, Armento M, Robertson S, Mullane C, Wolf N, et al. Cognitivebehavior therapy for depressed cancer patients in a medical care setting. Behav Ther 2008;39:126-36. doi: 10.1016/j.beth.2007.05.007

13. Shaheen Al Ahwal M, Al Zaben F, Khalifa DA, Sehlo MG, Ahmad RG, Koenig HG. Depression in patients with colorectal cancer in Saudi Arabia. J Psychosoc Oncol 2015;24:1043-50. doi: 10.1002/pon.3706

14. Hopko DR, Lejuez CW, LePage J, Hopko SD, McNeil DW. A brief behavioral activation treatment for depression: A randomized trial within an inpatient psychiatric hospital. Behav Modificat 2003; 27: 458-69. doi: 10.1177/0145445503255489

15. Jacobson NS, Martell CR, Dimigjian S. Behavioral activation treatment for depression: returning to contextual roots. Clin Psychol Sci Pract 2001; 8: 255-70. doi: 10.1093/ clipsy.8.3.255

16. Lejuez CW, Hopko DR, Hopko SD. A brief behavioral activation treatment for depression: Treatment manual. Behav Modificat 2001; 25: 255-86. doi: 10.1177/0145445501252005

17. Lejuez CW, Hopko DR, LePage J, Hopko SD, McNeil DW. A brief behavioral activation treatment for depression. Cogn Behav Pract 2001;8:164-75.

18. Ibañez E, Soriano J. Intervención psicológica en enfermos de cáncer: planteamientos del presente, deseos de futuro. Anál Modif Cond (Internet) 2008; 34:259-90.

19. Quintero MF, Finck C. Intervenciones psicológicas eficaces en pacientes con cáncer de mama en Latinoamérica y España: una revisión sistemática. Psicooncología 2018;15:4964. doi: 10.5209/PSIC.59174

20. Font A, Rodríguez E. Eficacia de las intervenciones psicológicas en mujeres con cáncer de mama. Psicooncología 2007;4: 423-46.

21. Moyer A, Sohl SJ, Knapp-Oliver S, Schneider S. Characteristics and methodological quality of 25 years of research investigating psychosocial interventions for cancer patients. Cancer Trea. Rev 2009;35:475-84. doi: 10.1016/j.ctrv.2009.02.003

22. Torre-Luque A, Gambara H, López E, Cruzado JA. Psychological treatments to improve quality of life in cancer context: A meta-analysis. Int J. Clin Health Psychol 2016;16:2119. doi: 10.1016/j.ijchp.2015.07.005

23. García Rasero AM, Tamayo Hernández JA Revisión sistemática sobre la eficacia de la Terapia de Aceptación y Compromiso (ACT) en el tratamiento psicológico de pacientes con cáncer. Psicooncología 2019;16:101-25. doi: 10.5209/PSIC.63651

24. Villoria E, Fernández C, Padierna C, González S. La intervención psicológica en pacientes oncológicos: una revisión de la literatura 2000-2014. Psicooncología, 2015;12:207-36.

25. Bianchi J, Henao A. Activación conductual y depresión: conceptualización, evidencia y aplicaciones en Iberoamérica. Ter Psicol 2015;33:69-80. doi:10.4067/S071848082015000200002 
26. Cullen JM, Spates CR, Pagoto S, Doran N. Behavioral activation treatment for major depressive disorder: a pilot investigation. Behav Anal Today 2006;7:151-66. doi: 10.1037/ h0100150

27. Hopko DR, Bell JL, Armento MEA, Hunt MK, Lejuez CW. Behavior therapy for depressed cancer patients in primary care. Psychother Theor Res Pract Train 2005;42:236-43. doi: 10.1037/0033-3204.42.2.236

28. Hopko DR, Lejuez CW, Hopko SD. Behavioral activation as an intervention for coexistent depressive and anxiety symptoms. Clin Case Stud 2004;3:37-48. doi: $10.1177 / 1534650103258969$

29. Hopko DR, Lejuez CW, Ruggiero KJ, Heifert GH. Contemporary behavioural activation treatments for depression: procedures, principles, and progress. Clin Psychol Rev 2003;23:699-717. doi: 10.1016/S0272-7358(03)00070-9

30. Becerra Gálvez AL, Reynoso Erazo L, Bravo González MC, Ordaz Carrillo MI. Activación conductual para el control de la depresión en pacientes oncológicos: una revisión. Psicooncología 2017;14:203-16. doi: 10.5209/PSIC.57080.

31. Hopko DR, Armento ME, Robertson SM, Ryba MM, Carvalho JP, Colman LK, et al. Brief behavioural activation and problem-solving therapy for depressed breast cancer patients: randomized trial. J Consult Clin Psychol 2011;70:834-49. doi: 10.1037/a0025450

32. Hopko DR, Clark CG, Cannity K, Bell JL. Pretreatment depression severity in breast cancer patients and its relation to treatment response to behavior therapy. Health Psychol 2016;35:10-8. doi: 10.1037/hea0000252

33. Hopko DR, Funderburk JS, Shorey RC, McIndoo CC, Ryba MM, File AA et al. Behavioral activation and problem-solving therapy for depressed breast cancer patients: preliminary support for decreased suicidal ideation. Behav Modificat 2013;37:747-67. doi: $10.1177 / 0145445513501512$

34. Hopko DR, Robertson SM, Carvalho JP. Sudden gains in depressed cancer patients treated with behavioral activation therapy. Behav Ther 2009;40:346-56. doi: 10.1016/j. beth.2008.09.001.

35. Zigmond AS, Snaith RP. The Hospital Anxiety and Depression Scale. Acta Psychiatr Scand 1983;67:361-70

36. Cabrera V, Martín-Aragón M, Terol M, Núñez R, Pastor M. La Escala de Ansiedad y Depresión Hospitalaria (HAD) en fibromialgia: Análisis de sensibilidad y especificidad. Ter Psicol 2015;33:181-93.

37. Iani L, Lauriola M, Constantini M. A confirmatory bifactor analysis of the Hospital Anxiety and Depression Scale in an Italian community sample. Health Qual Life Outcomes 2014;5:84. doi: 10.1186/1477-7525-12-84

38. Terol, MC, Cabrera V, Martín M. Revisión de estudios de la Escala de Ansiedad y Depresión Hospitalaria (HAD) en muestras españolas. Anal Psicol 2015;31:494-503. doi: 10.6018/analesps.31.2.172701

39. Villoria E, Lara L. Assessment of the Hospital Anxiety and Depression Scale for cancer patients. Rev Medica Chile 2018;146: 300-07. doi: 10.4067/s0034-98872018000300300

40. Villoria E, Fernández, C. (dir). Terapia de activación conductual para la prevención de los trastornos emocionales y la promoción de la calidad de vida en pacientes oncológicos [tesis doctoral no publicada]. [Oviedo]: Universidad de Oviedo; 2012, 567 p.

41. Forster S, Marsh EJ. Assessing social validity in clinical treatment research: issues and procedures. J Consult Clin Psychol 1999;67:308-19. doi: 10.1037/0022-006x.67.3.308 
42. Biglia N, Moggio G, Peano E, Sgandurra P, Ponzone R, Nappi RE et al. Effects of surgical and adjuvant therapies for breast cancer on sexuality, cognitive functions, and body weight. J. Sex. Med. 2010;7:1891-900. doi: 10.1111/j.1743-6109.2010.01725.x

43. Farthmann J, Hanjalic-Beck A, Veit J, Rautenberg B, Stickeler E, Erbes T, et al. The impact of chemotherapy for breast cancer on sexual function and health-related quality of life. Support. Care Cancer 2016; 24:2603-09. doi:10.1007/s00520-015-3073-2.

44. Tiezzi MF, de Andrade JM, Romão AP, Tiezzi DG, Lerri MR, Carrara HA, et al. Quality of life in women with breast cancer treated with or without chemotherapy. Cancer Nurs 2017;40: 108-16. doi: 10.1097/NCC.0000000000000370.

45. Fernández C, Amigo I, Villoria E, Padierna C, Fernández R, Peláez I. Influencia del estado emocional en la sintomatología referida por pacientes con cáncer de mama y cáncer de pulmón durante el tratamiento con quimioterapia. Medicina Paliativa 2013;20:85-92. doi: 10.1016/j.medipa.2012.05.002

46. Krebber AM, Buffart LM, Kleijn G, Riepma IC, de Bree R, Leemans CR, et al. Prevalence of depression in cancer patients: a meta-analysis of diagnostic interviews and self-report instruments. Psychooncology 2014;23:121-30. doi: 10.1002/pon.3409.

47. de Vries M, Stiefel F Psycho-oncological interventions and psychotherapy in the oncology setting. Recent Results Cancer Res 2014;197:121-35. doi: 10.1007/978-3-642-40187-9_9.

48. Watts S, Prescott P, Mason J, McLeod N, Lewith G. Depression and anxiety in ovarian cancer: a systematic review and meta-analysis of prevalence rates. BMJ Open 2015; 5(11): e007618.. doi: 10.1136/bmjopen-2015-007618.

49. Fallowfield L. Treatment decision-making in breast cancer: the patient-doctor relationship. Breast Cancer Res Treat 2008;112:5-13. doi: 10.1007/s10549-008-0077-3

50. González S, Fernández C, Mota MJ, García P, Pedrosa I, Pérez M. Estado emocional y flexibilidad psicológica en sobrevivientes de cáncer de mama. Eur J Oncol Nurs 2017;30:75-83. doi: 10.1016/j.ejon.2017.08.006

51. Martínez López P, Andreu Vaillo Y, Galdón Garrido MJ, Romero Retes R, García-Conde Benet A, Llombart Fuertes P. Distrés emocional y problemas asociados en población oncológica adulta. Psicooncología 2017;14:217-28. doi: 10.5209/PSIC.57081

52. Banks E, Byles JE, Gibson RE, Rodgers B, Latz IK, Robinson IA, Williamson AB, et al. Is psychological distress in people living with cancer related to the fact of diagnosis, current treatment or level disability? Findings from a large Australian study. Med J Aust 2010;193:62-7. doi: 10.5694/j.1326-5377.2010.tb03931.x

53. Padierna C, Fernández C, Amigo I, Gracia J M, Fernández R, Peláez I, et al. Estudio longitudinal de los parámetros de calidad de vida en pacientes oncológicos. Psicooncología 2004;1: 191-204.

54. Fawcy FI. Psychosocial interventions for patients with cancer: What works and what doesn't. Eur J Cancer 1999;35:1559-64. doi: 10.1016/S0959-8049(99)00191-4

55. So WKW, Marsh G, Ling WM, Leung FY, Lo JCK, Yeung M, et al. Anxiety, depression y quality of life among Chinese breast cancer patients during adjuvant therapy. Eur J Oncol Nurs 2010;14:17-22. doi: 10.1016/j.ejon.2009.07.005

56. Lauver DR, Connolly-Nelson K, Vang P. Stressors and coping strategies among female cancer survivors after treatments. Cancer Nurs 2007;30:101-11. doi: 10.1097/01. NCC.0000265003.56817.2c

57. Navarro E, Limonero JT, Maté J, Gómez-Romero MJ. Necesidades de comunicación e información en el paciente oncológico superviviente. Psicooncología 2010;7:127-41.

58. Sammarco A, Konecny LM. Quality of life, social support and uncertainty among Latin breast cancer survivors. Oncol Nurs Forum 2008;35:844-49. doi: 10.1188/08.ONF.844-849 